\title{
Lunar Tides and the Long-Term Variation of the Peak Latitude Anomaly of the Summer Sub-Tropical High Pressure Ridge over Eastern Australia
}

\author{
Ian R.G. Wilson*
}

\author{
Queensland Department of Education and Training, Toowoomba, QLD, Australia
}

\begin{abstract}
This study looks for evidence of a correlation between long-term changes in the lunar tidal forces and the interannual to decadal variability of the peak latitude anomaly of the summer (DJF) subtropical high pressure ridge over Eastern Australia $\left(\mathrm{L}_{\mathrm{SA}}\right)$ between 1860 and 2010. A simple "resonance" model is proposed that assumes that if lunar tides play a role in influencing $\mathrm{L}_{\mathrm{SA}}$, it is most likely one where the tidal forces act in "resonance" with the changes caused by the far more dominant solar-driven seasonal cycles. With this type of model, it is not so much in what years do the lunar tides reach their maximum strength, but whether or not there are peaks in the strength of the lunar tides that re-occur at the same time within the annual seasonal cycle. The "resonance" model predicts that if the seasonal peak lunar tides have a measurable effect upon $\mathrm{L}_{\mathrm{SA}}$ then there should be significant oscillatory signals in $\mathrm{L}_{\mathrm{SA}}$ that vary in-phase with the 9.31 year draconic spring tides, the 8.85 year perigean spring tides, and the 3.80 year peak spring tides. This study identifies significant peaks in the spectrum of $\mathrm{L}_{\mathrm{SA}}$ at $9.4(+0.4 /-0.3)$ and $3.78( \pm 0.06)$ tropical years. In addition, it shows that the 9.4 year signal is in-phase with the draconic spring tidal cycle, while the phase of the 3.8 year signal is retarded by one year compared to the 3.8 year peak spring tidal cycle. Thus, this paper supports the conclusion that long-term changes in the lunar tides, in combination with the more dominant solar-driven seasonal cycles, play an important role in determining the observed inter-annual to decadal variations of $\mathrm{L}_{\mathrm{SA}}$.
\end{abstract}

Keywords: Atmospheric pressure; high pressure ridge; lunar tides.

\section{INTRODUCTION}

According to current tidal theories (Chapman and Lindzen [1]; Lindzen [2]), atmospheric tides are excited primarily by the Sun's heating of the atmosphere, whereas ocean tides are excited primarily by the Moon's gravitational tidal pull. This means that short-term atmospheric tides are detected as regular but small oscillations in surface pressure with periods of 12 hours $\left(\mathrm{S}_{2}\right)$ and 24 hours $\left(\mathrm{S}_{1}\right)$ that are related to the solar day. In contrast, ocean tides are primarily detected as oscillations in sea-surface height with semidiurnal $\left(\mathrm{M}_{2}=12.42\right.$ hours $)$ and diurnal $\left(\mathrm{M}_{1}=24.85\right.$ hours $)$ periods that are related to the longer lunar day (i.e. the time between successive lunar transits). Hence, it is generally believed that any atmospheric tides that are induced by lunar tidal forces are far too small to have any noticeable effect upon atmospheric circulation in the Earth's lower atmosphere, leaving solar radiation as the dominant external forcing factor.

However, Li [3], Li and Zong [4] and Li et al. [5] have shown that, contrary to currently accepted tidal theories, cyclical changes in lunar tidal forcing produce 27.3 day and 13.6 day periodic atmospheric tides. They detect these atmospheric tides in the tropical troposphere at heights above the $700 \mathrm{hPa}$ isobaric surface $(\sim 3000 \mathrm{~m})$. Furthermore, Li et al. [5] claim that the changes in lunar tidal forcing that are

*Address correspondence to this author at the Queensland Department of Education and Training, Toowoomba State High, Mt. Lofty Campus, Stuart St., Toowoomba, QLD 4350, Australia; Tel: +61 746371 363; Fax: +61 7 46371 310; E-mail: irgeo8@bigpond.com responsible for the lunar-driven atmospheric tides, are the same as those that are collectively responsible for the periodic changes in the Earth's length-of-day (LOD), on fortnightly to seasonal timescales.

Periodic variations in the LOD caused by tidal deformation of the polar moment of inertia (Defraigne and Smits [6]; Yoder et al. [7]) reveal that, on fortnightly to seasonal timescales, there are five significant periodic variations in the LOD that are caused by the lunar tides. These tidal variations have periods at 13.63 days (Mf'), 13.66 days (Mf), 14.77 days (Msf), 27.56 days (Mm), and 182.62 days (Ssa) (Varga et al. [8]).

Thus, if you accept the claim of Li et al. [5] that their atmospheric tides exhibit the same periodicities as those found in the Earth's LOD, it opens up the possibility that these short-term variations in the LOD could be used to determine the periods for tidally-induced long-term variations in Earth's atmospheric pressure distribution.

The simplest way to determine the possible periods for atmospheric tides on inter-annual to decadal time scales (i.e. from 2 to 20 years) is to list the beat frequencies of the tidally-induced fortnightly and monthly variations in the LOD. There are three possible beat frequencies that lie between 2 and 20 years. These are:

3.00/6.00 years: The 6.0 year period corresponds to the time required for the perigee of the lunar orbit to realign with the same node of the lunar orbit. Of course, this means that the fundamental repetition period is actually $\mathbf{3 . 0 0}$ years since the lunar orbit has two distinct nodes. 
8.85 years: This period corresponds to the time required for the line-of-apses of the lunar orbit to precess once around the Earth with respect to the stars.

9.30/18.6 years: The 18.6 year period corresponds to the time required for the line-of-nodes of the lunar orbit to precess once around the Earth with respect to the stars. Again, this implies a fundamental repetition period of $\mathbf{9 . 3 0}$ years because of the lunar orbit's two nodes.

The problem with this simple analysis is that it does not take into account the different ways in which the lunar tides can interact with the Earth's climate system. The first aim of this work is to determine which of these tidal periods are most likely to be observed for the long-term atmospheric tides, given some simple constraints upon the way in which the lunar tides interact with the Earth's climate system. The second aim is to extend the pioneering work of Li et al. [5] by looking for evidence of atmospheric tides on inter-annual to decadal timescales. In particular, the paper will investigate if there is any link between the variations in the strength of the lunar tides and the atmospheric pressure variations over Eastern Australia.

The methodology used in this study is described in section 2. The first part of section 2 outlines the properties of the lunar tides that determine the periods of long-term atmospheric tides. The second part of section 2 presents the main data set used in this study. This data set consists of a time series of the summer (DJF) anomaly of the peak latitude of the sub-tropical high pressure ridge over Eastern Australia $\left(\mathrm{L}_{\mathrm{SA}}\right)$, for each year from 1860 to 2010 . The third part of section 2 describes the different methods of spectral analysis that are used to identify oscillatory signals in the spectrum of the data set.

The main results of this investigation, along with the results of the spectral analysis of the principle data set, are presented in section 3. Finally, the conclusions are outlined in section 4 .

In conclusion, it is important to note that many of the time intervals and orbital periods that are quoted in this paper are means or averages. The fact that they are averages allows us to quote them to the level of precision stated. However, in many cases there can be significant variation of these time intervals and orbital periods about the quoted value of the mean. Hence, the average values only become meaningful if we are considering time intervals that are long compared to the orbital period of the Moon.

\section{METHODOLOGY}

\subsection{The Potential Periods for Long-Term Atmospheric Tides}

There are two methods that can be used to work out the possible periods for long-term atmospheric tides. The first method assumes that lunar-tidal forces act independently of the other forcing factors that produce significant long-term variations in atmospheric pressure (e.g. seasonal variations in solar heating). Under this assumption, you would expect to see long-term periodicities in the pressure records that would match periodicities of the most extreme peak lunar tides. The second method investigates what happens when this assumption breaks down.
The most significant large-scale systematic variations of the atmospheric surface pressure, on an inter-annual to decadal time scale, are those caused by the seasons. These variations are predominantly driven by changes in the level of solar insolation with latitude that are produced by the effects of the Earth's obliquity and its annual motion around the Sun. This raises the possibility that the lunar tides could act in "resonance" with (i.e. subordinate to) the atmospheric pressure changes caused by the far more dominant solardriven seasonal cycles. With this type of simple "resonance" model, it is not so much in what years do the lunar tides reach their maximum strength, but whether or not there are peaks in the strength of the lunar tides that re-occur at the same time within the annual seasonal cycle.

Hence, a strong peak in the lunar tidal forces that slowly drifts through the seasons, with each advancing year, may not be as effective at influencing the atmospheric pressure distribution, as a weaker tidal peak that appears at precisely the same time during the seasonal (tropical) year (hereafter referred to as the seasonal peak tides).

\subsubsection{The Most Extreme Peak Lunar Tides}

Keeling and Whorf [9] has shown that the most extreme peak lunar tides occur whenever the Sun and Moon are in direct mutual alignment. This occurs at syzygy (i.e. either Full or New Moon), when there is either a lunar or solar eclipse, the Moon is at perigee, and the Earth is at perihelion. According to these authors, the dominant periodicities for exceptional peak tides should matched the shortest period over which there is a re-occurrence of syzygy, an eclipse, perigee and perihelion. This repetition period is the 18.03 tropical year Saros cycle and its immediate sub-multiples (i.e. 3.01, 6.01, and 9.02 tropical years). Column one of Table 1 summarizes the periodicities expected for the most extreme peak lunar tides.

\subsubsection{The Seasonal Peak Tides}

There are four factors which can affect the strength of seasonal peak tides. The first is the proximity of the Earth/Moon system to the Sun. The second is the relative position of the Moon with respect to the Sun. The third is the proximity of a New/Full Moon to one of the nodes of the lunar orbit. Finally, the fourth is the proximity of a New/Full Moon to the perigee of the lunar orbit.

\subsubsection{The Proximity of the Earth/Moon System to the Sun}

Due to the elliptical nature $(e=0.0167)$ of the Earth's orbit, the distance of the Earth/Moon system from the Sun varies between an aphelion of 152.1 million $\mathrm{km}$ around July $04^{\text {th }}$ to a perihelion of 147.1 million $\mathrm{km}$ on January $3^{\text {rd }}$ (Standish and Williams [10]). This means that the strength of lunar tidal forces near January $03^{\text {rd }}$ are noticeably enhanced compared to those that are near July $04^{\text {th }}$. Hence, the effects of any long-term seasonal peak tides upon atmospheric pressure will be naturally enhanced if these peak tides are aligned with the date of perihelion.

2.1.2.2. The Relative Position of the Moon with Respect to the Sun

Higher than normal spring tides occur once every semisynodic month (Msf), whenever the Sun, Earth and Moon are co-aligned at either New or Full Moon. It turns out that 
12.5 synodic months are 3.890171 days longer than one tropical year (N.B. from this point forward, the word "year" will mean one tropical or seasonal year $=365.2421897$ days (J2000) (McCarthy and Seidelmann [11], unless indicated). Hence, if a spring tide occurs on a given day of the year, 3.796 tropical years will pass before another spring tide occurs on the same day of the year. This occurs because:

$(0.5$ synodic months $) /(12.5$ synodic months - tropical year $)=$ $(14.7652944$ days/3.890171 days $)=3.796$ years.

In addition, it can be shown that multiples of half of the lunar synodic cycle (Msf) are almost exactly equal to whole multiples of a year, for 4.0 years, $4.0+4.0=8.0$ years, $4.0+$ $4.0+3.0=11.0$ years, $4.0+4.0+3.0+4.0=15.0$ years, and $4.0+4.0+3.0+4.0+4.0=19.0$ years.

Hence, spring tides that occur on roughly the same day of the year follow a 4:4:3:4:4 year spacing pattern (with an average spacing of $(4+4+3+4+4) / 5=3.8$ years $)$, with the pattern repeating itself after a period of almost exactly 19 years. The 19.0 year period is known as the Metonic cycle. This cycle results from the fact that 235 Synodic months = 6939.688381 days $=19.000238$ Tropical years.

Finally, the variation in strength of the seasonal peak spring tides from year to year is best measured by the number of days that the New/Full Moon is from the date of Perihelion (i.e. January $03^{\text {rd }}$ ).

2.1.2.3. The Proximity of the New/Full Moon to One of the Nodes of the Lunar Orbit

The Moon moves around the Earth in an elliptical orbit that is inclined to the plane of the ecliptic by $\sim 5.1^{\mathrm{O}}$ (Allen and Cox [12]). This means that the Moon crosses the ecliptic at two points known as the nodes of the lunar orbit. Hence, stronger than normal spring tides (known as draconic spring tides) occur whenever a New/Full Moon takes place near one of the nodes of the lunar orbit.

The Moon moves from one node back to the same node once every 29.212221 days (J2000) (Chapront-Touzé and Chapront, [13]). This period of time is called the Draconic or Nodal lunar month. It turns out that 13.5 draconic months are 2.122791 days longer than one year (Chapront-Touzé and Chapront [13]). Hence, if a lunar node aligns with the Sun on a given day of the year, 6.410 years will pass before another lunar node aligns with the Sun on roughly the same day of the year. This occurs because:

(0.5 draconic months)/(13.5 draconic months - tropical year) $=(13.606110$ days $/ 2.122791$ days $)=6.410$ years .

Unfortunately, when a lunar node realigns with the Sun on roughly the same day of the year, the Moon is no longer at the same lunar phase. In order to have a lunar node realign with the Sun on the same day of the year, and for the Moon to return to the same phase (e.g. New/Full Moon) as well, it would take a period of time set by the beat period between 3.796 and 6.410 years i.e. 9.308 years. This means that if a New Moon takes place when one of the lunar nodes points at the Sun, 9.31 years later, a Full Moon will occur when a lunar node points at the Sun. Thus, the spacing between draconic spring tides is 9.31 years, a period equal to half of the 18.61336 year draconic lunar cycle $(=9.307$ years $)$.
Technically speaking, draconic spring tides do not fall exactly on the same day of the annual seasonal cycle. However, they do take place within $+5 /-4$ days either side of a given date (with an average absolute difference of only 2.6 days), so they can be considered to be quasi-peak seasonal tides that take place on roughly the same day of the year, once every 9 or 10 years.

Finally, the variation in strength of draconic seasonal peak tides from year to year is best measured by the angle (in degrees) between the lunar line-of-nodes and the Earth-Sun axis, at the time of perihelion.

\subsubsection{The Proximity of the New/Full Moon to the Perigee of the Lunar Orbit}

The distance of the Moon from the Earth varies from a minimum (perigee) of $356,500 \mathrm{~km}$ to a maximum (apogee) of $406,700 \mathrm{~km}$ (Meeus [14]). Hence, stronger than normal spring tides occur whenever a New/Full Moon take places at the point of closest perigee in the lunar orbit.

The Moon moves from one perigee to the next in 27.554551 days (J2000) (Chapront-Touzé and Chapront [13]), a period of time known as one anomalistic month. It turns out that 13.5 anomalistic months are 6.7442337 days longer than one tropical year. Hence, if a lunar perigee aligns with the Sun on a given day of the year, 2.043 years will pass before lunar apogee aligns with the Sun on the same day of the year. This occurs because:

(0.5 anomalistic months)/(13.5 anomalistic months - tropical year $)=(13.777275$ days $/ 6.7442337$ days $)=2.043$ years .

Unfortunately, when a lunar apogee realigns with the Sun on the same day of the year, the Moon is no longer at the same lunar phase. In order to have lunar apogee realign with the Sun on the same day of the year, and for the Moon to return to the same phase as well (so that the opposite lunar phase occurs at lunar perigee), it would take a period set by the beat period between 3.796 and 2.043 years $=4.424$ years. This period of time is one half of the 8.8506 year lunar apse cycle $(=4.425$ tropical years $)$.

These enhanced spring tides qualify as quasi-seasonal peak tides for the simple reason that they re-occur on roughly the same day of the year once every 4 or 5 years, in a sequence that repeats itself every 31 years e.g. $(5+5+4+$ $4+5+4+4) / 7=4.429$ years. The reason for the 31.00686 year repetition period is the fact that 383.5 synodic lunar months $=11324.980825$ days is almost exactly equal to 411.0 anomalistic lunar months $=11324.92000$ days. The difference between these two cycles is very small, amounting to only 1.46 hours over the 31 years. There is also another near coincidence in that 27.5 anomalistic lunar years $=11324.071832$ days, ensuring that if a New Moon occurs at closest perigee, 31 Tropical years later, at almost exactly the same time of the year, a Full Moon will occur at closest perigee.

It is important to remember, however, that the enhanced spring tides that take place when a New Moon is at closest perigee (also known as perigean spring tides (Woods [15]) produce significantly stronger tides, than the enhanced spring tides that take place when Full Moon is a closest perigee. This means that the peak seasonal tidal cycle for perigean spring tides should have a period of 8.85 years, 
rather than 4.43 years. Column two of Table 1 summarizes the periodicities that are expected to be produced by the different types of seasonal peak tides.

Finally, the variation in strength of perigean spring tides from year to year is best measured by the angle (in degrees) between the lunar line-of-apse and the Earth-Sun axis, at the time of perihelion.

Table 1. The Predicted Periods for Tidally-Induced Atmospheric Tides on Inter-Annual to Decadal Time Scales

\begin{tabular}{|c|c|}
\hline $\begin{array}{c}\text { The Periods for Extreme } \\
\text { Peak Tides (Years) }\end{array}$ & $\begin{array}{c}\text { The Periods for Peak } \\
\text { Seasonal Tides (Years) }\end{array}$ \\
\hline \hline 3.01 & 3.80 \\
\hline 6.01 & 8.85 \\
\hline 9.02 & 9.31 \\
\hline 18.03 & 18.61 \\
\hline
\end{tabular}

\subsection{Data}

There are three reasons why the peak latitude of the SubTropical (High Pressure) Ridge over Eastern Australia (STR) is well suited for investigating the potential influence of lunar tidal forces upon the atmosphere.

Firstly, the surface atmospheric pressure records are continuously available from 1850 to 2010 . This means that the record length is long enough to permit a viable search for a tidal signal that extends over the inter-annual to decadal time scales that are normally associated with long-term lunar tidal variations.

Secondly, the STR provides us with a single parameter that describes the broad changes in atmospheric pressure over a relatively large geographic region. This allows us to search for the large scale spatial changes in atmospheric pressure that are likely to be associated with the effects of variations in the long-term lunar tidal forces.

Finally, the STR has very important climate implications for Eastern Australia. Pittock [16] has shown that the interannual variability of the latitude of the surface subtropical high pressure maximum over Eastern Australia is major mechanism influencing inter-annual rainfall variability. It has also been shown to be connected to the inter-annular variability of the annual mean maximum temperatures (Coughlan [17]), zonal westerly winds (Thresher [18]), meridional wind and mean air temperature, and ozone (Pittock [19]).

The UK Met Office Hadley-Centre (UKMO) has published a data set called hadSLP2r.asc (Adam and Ansell [20]; www.hadobs.org [21]) that contains the mean monthly sea-level pressure (MSLP), averaged over 5 x 5 degree latitude-longitude bins, between the years (January) 1850 to (June) 2010. The hadSLP2r data has been used to create a meridional profile of the MSLP, for each of the summer months (i.e. December, January and February) for the years 1852 to 2010 (hereafter referred to as the UKMO data set). This has been done by taking a latitudinal average of three 5 x 5 degree bins centered at $140 \mathrm{E}, 145 \mathrm{E}$, and $150 \mathrm{E}$, for each 5 degree step in latitude between 0 and 65 degrees south. N.B. the profile data points have not been weighted to correct for the difference in area between $5 \times 5$ degree bins with changing latitude.

Fig. (1) shows a meridional profile of the MSLP for February 1984. This profile is a typical example of the meridional profiles found in the UKMO data set. In this profile, we can see a zone of low pressure produced by the Summer Monsoonal Trough centred near $10^{\circ} \mathrm{S}$, a ridge of high pressure produced by the STR near $40^{\circ} \mathrm{S}$, and a second zone of low pressure south of $60^{\circ}$ that is associated with the Sub-Polar Trough.

Cubic spline functions (DADisp - DSP Development Corporation, [22]) have been fitted to all of the monthly meridional profiles in the UKMO data set. These cubic spline fits have then been used to locate the monthly peak latitude of the STR (hereafter referred to as L) through a process of interpolation.

The process of interpolation has been carried out using cubic spline curves to obtain (interpolated) MSLP measurements, spaced in steps of $1 / 2$ a degree, between each of the observed values of MSLP. These measurements are used to locate the maximum (interpolated) MSLP of the meridional profile, and then a ruler, along with eyeestimations of the fitted curves, are used to determine L, to the nearest 0.1 degree.

In addition, Bezier functions (Microsoft Excel software package - Microsoft Office 2007 [23]) have been fitted to all of the monthly meridional profiles to obtain values of $\mathrm{L}$ through a process of interpolation. However, in this case, only a ruler and eye-estimations of the fitted Bezier curves have been used to determine $\mathrm{L}$, to the nearest 0.1 degree.

The use of two distinctly different methods of interpolation on the same set of data allows us to estimate the error associated with the interpolation process. The error in $\mathrm{L}$ due to the process of interpolation is equivalent to the sample mean of the absolute difference between corresponding measurements that are derived from the two methods of interpolation. A comparison of the two data sets shows that the error for each measurement of $\mathrm{L}$ due to process of interpolation is approximately 0.17 degrees. Hence, if we adopt a value of $0.1 \mathrm{deg}$ for the instrumental error, each measurement of $\mathrm{L}$ should have an overall error $(\Delta \mathrm{L}) \sim 0.3$ degrees.

Monthly anomalies for $\mathrm{L}$ were obtained using a mean monthly value of L for the base period 1961-1990 (William and Stone [24]). William and Stone [24] point out that it is important to investigate the monthly anomaly of $\mathrm{L}$ on a seasonally-averaged, rather than annually-averaged basis. Following their advice, we have taken the latitude anomalies for December, January and February for each year and averaged them together to give a mean summer value for the anomaly of $\mathrm{L}$ (hereafter referred to as $\mathrm{L}_{\mathrm{SA}}$ ) for all of the years from 1851 to 2010 . $\mathrm{L}_{\mathrm{SA}}$ is defined so that a positive value means that the STR is north of the mean latitude for that summer season

Fig. (2) shows $L_{S A}$ for the years from 1851 to 2010 . The first feature to note about the data plotted in this figure is that the bulk of the $\left(\mathrm{L}_{\mathrm{SA}}\right)$ values lie between \pm 1.0 degree (the 


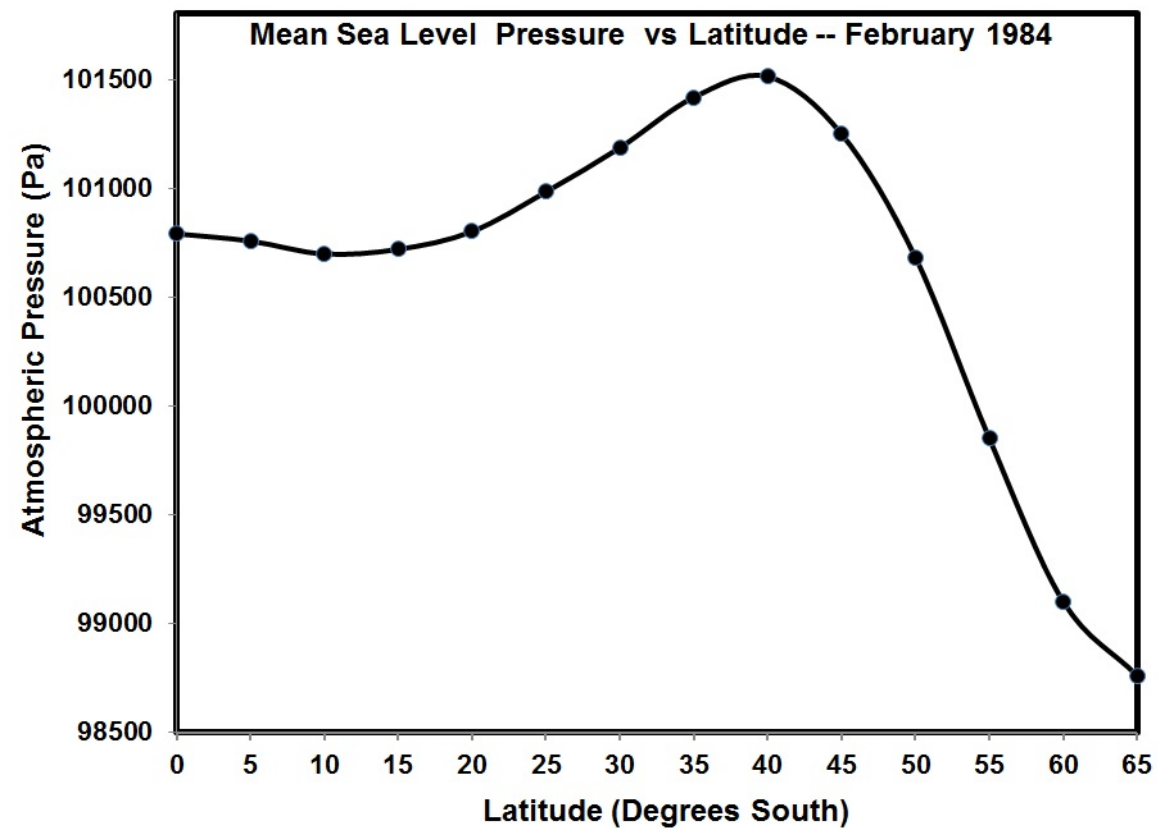

Fig. (1). A profile of the mean sea level pressure versus latitude for February 1984.

standard deviation of the full data set is 0.93 degrees) with a very stable long term mean close to +0.28 degrees (N.B. the mean of the data set plotted in Fig. (2) has been set to zero by subtracting 0.28 degrees from all of the values of $\mathrm{L}_{\mathrm{SA}}$ ). The one noticeable exception to this long-term pattern is an abrupt upturn in the $\left(\mathrm{L}_{\mathrm{SA}}\right)$ data prior to 1856 . The first five years of the data set (i.e. 1851 to 1855) has a mean that differs from the plotted long-term mean of zero by +1.65 degrees. The magnitude of this step-like deviation is almost three times the measurement error for $\left(\mathrm{L}_{\mathrm{SA}}\right)$ (i.e. 0.6 degrees) and almost two times the data's standard deviation of 0.88 degrees, between 1860 and 2010. This raises the possibility that there may be a systematic error in the first five years worth of hadSLP2r MSLP measurements.

Given that there is some uncertainty as to whether this abrupt deviation in the first five years of the data is a genuine

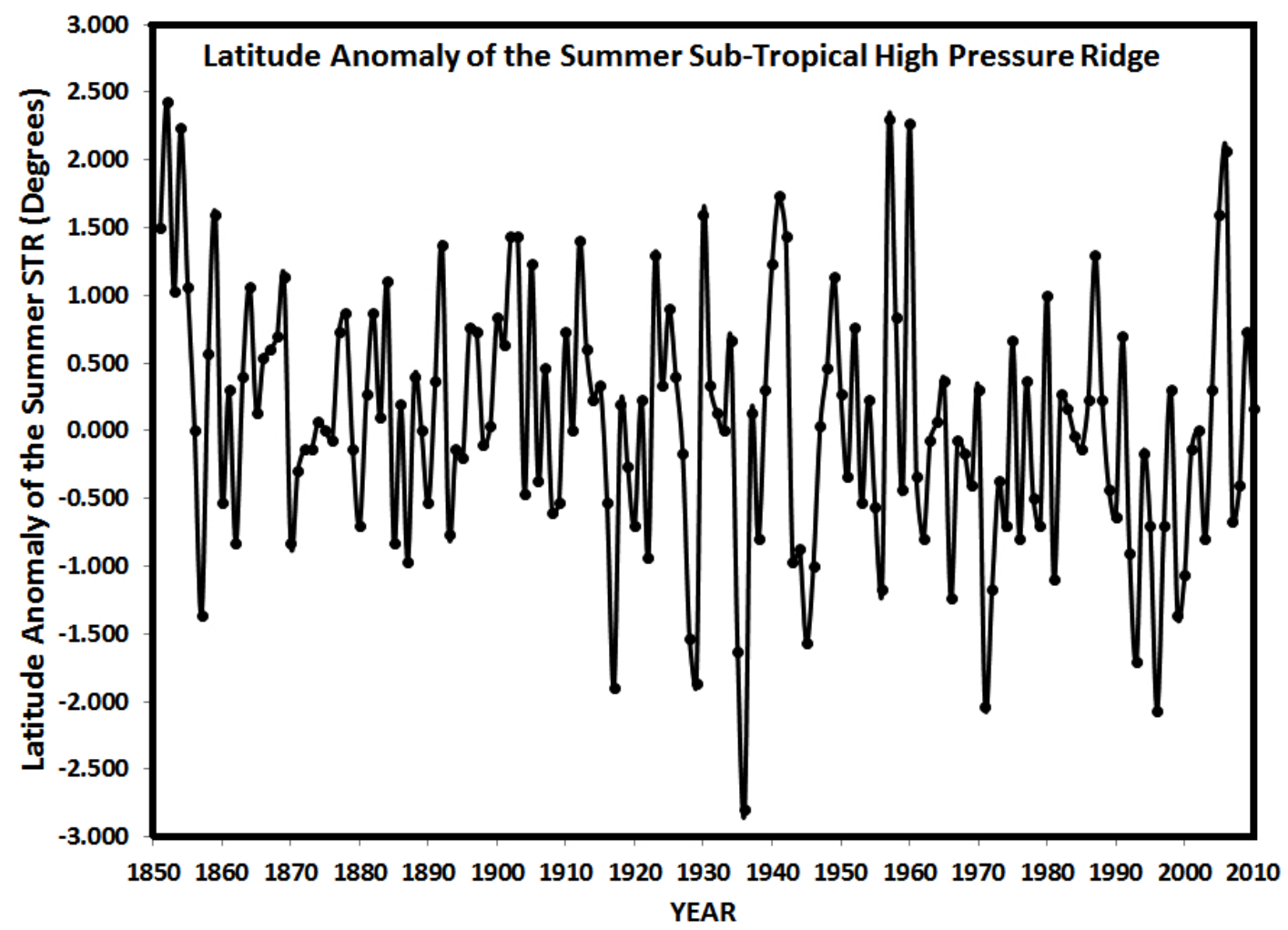

Fig. (2). The anomaly of the peak latitude of the Summer Sub-Tropical High Pressure Ridge over Eastern Australia ( $\left.\mathrm{L}_{\mathrm{SA}}\right)$ for the years from 1851 to 2010 . 
trend, as opposed to a flaw in the original MSLP measurements, all $\left(\mathrm{L}_{\mathrm{SA}}\right)$ values prior to 1860 will be left out of the final data set. This has been done in order to err on the side of caution.

\subsection{Methods}

\subsubsection{Least Squares Spectral Analysis - LSSA}

The Lomb-Scargle periodogram is a least squares spectral analysis technique that is a very powerful method for finding and testing the significance of weak periodic signals ([Lomb [25]; Scargle [26], [27]). It is specifically designed to work with unevenly spaced data but there is no reason why it cannot be used with data that is evenly spaced. The Lomb-Scargle periodogram can test if peaks in the spectrum of $\mathrm{L}_{\mathrm{SA}}$ are significant compared to a red-noise background, formed by a first order auto-regressive (AR1) process (Schulz and Mudelsee [28]). Another additional advantage of the Lomb-Scargle periodogram is that it can be used to test whether or not the use of an AR1 red-noise model is consistent with the $\mathrm{L}_{\mathrm{SA}}$ spectrum.

A program called Redfit 3.8e (Windows XP version (Schulz and Mudelsee [28]) is used to generate a LombScargle periodogram of the $\mathrm{L}_{\mathrm{SA}}$ data set, and then the resulting periodogram is displayed in order to determine if there are any narrow-band spectral features that are significant.

\subsubsection{Singular Spectrum Analysis - SSA}

Singular spectrum analysis (SSA) can be used to distinguish the contributions of oscillatory parts of a time series $\mathrm{L}_{\mathrm{SA}}$ from the parts that are associated with the longterm trends and noise. Additionally, this technique can be used to determine the times period(s) over which a particular oscillatory signal is most prominent.

The results obtained from the SSA method depend upon the lag window length (LW) that is chosen to deconstruct the time series. The larger the value of LW, the better the SSA technique is at distinguishing between different spectral features that are embedded in the time series. However, it is important that the value of LW not exceed half the total number of data points in the time series data. These guidelines, along with a process of trial and error, were used to determine a value of LW that was sufficiently large enough to distinguish the main periodic signals from the long-term trends and background noise of the spectrum (Caterpillar v1.0 1997 [29]).

Finally, the Caterpillarv1.0 program was used to reconstruct the oscillatory parts of the $\mathrm{L}_{\mathrm{SA}}$ time series that are associated with the significant features found in the spectrum of $\mathrm{L}_{\mathrm{SA}}$.

\subsubsection{Cross - Correlation Analysis - CCA}

In order to be able to claim a link between an oscillatory peak in the spectrum of $\mathrm{L}_{\mathrm{SA}}$ and one of the seasonal peak tides, it is necessary to show that both the period and phase of the oscillatory peak matches that expected for one of the seasonal peak tides.

A function called XCORR in a program called DADiSP [22] is used to cross-correlate $\mathrm{L}_{\mathrm{SA}}$ with two parameters that give a direct measure of the strength of the seasonal peak tides. The resulting corelograms are displayed in order to determine the phase-shift between oscillatory signals in the spectrum of $\mathrm{L}_{\mathrm{SA}}$ and their respective oscillatory signals in the seasonal peak tides.

\section{RESULTS}

\subsection{Least Squares Spectral Analysis}

A program called Redfit 3.8e (Schulz and Mudelsee [28]) was used to generate a Lomb-Scargle periodogram of the $\mathrm{L}_{\mathrm{SA}}$ data set. The parameters used in the configuration file needed to run Redfit were set to values that maximize the spectral resolution of the periodogram (N.B. for a detailed description of the parameters used with Redfit see Schulz and Mudelsee [28]).

The resulting spectrum is displayed in Fig. (3). The output of Redfit program indicates that the noise in the periodogram is consistent with an AR1 (red-noise) process. The solid continuous dark line running across the top of the spectrum in Fig. (3) is the critical false alarm level (CFAL) (Thomas [30]). Any periodic signals that have peak amplitudes exceeding this threshold level are believed to be inconsistent with an AR1 origin and so are considered significant. Hence, the only significant peaks in the spectrum in Fig. (3) are those at $9.4(+0.4 /-0.3)$ and $3.78( \pm 0.06)$ (N.B. the errors of the periods given are set at \pm half of the $6 \mathrm{~dB}$ bandwidth). The 9.4 year peak is consistent with the period of the 9.3 year draconic spring tidal cycle and the 3.8 year peak with the 3.8 year period of the spring tidal cycle.

\subsection{Singular Spectrum Analysis}

The Caterpillar v1.0 program was used to deconstruct the $\mathrm{L}_{\mathrm{SA}}$ time series into its principle components using a window length value (LW) of 45 (years). This value was chosen as it did not exceed half the total number of data points in the $\mathrm{L}_{\mathrm{SA}}$ time series, and it was sufficiently large enough to distinguish the main periodic signals from the long-term trends and background noise of the spectrum (Caterpillar v1.0 1997 [27]).

Fig. (4a) shows the time series that is reconstructed using the first two principal components (PC) of the SSA. Fig. (4b) shows the time series that is reconstructed from PC5 and 6. PC1 and 2 are associated with 9.3 year spectral feature, PC5 and 6 with the 3.8 year feature, and PC3 and 4 with the 2.6 year feature. Additionally, Fig. $(\mathbf{4 a}, \mathbf{b})$ indicate that the two dominant spectral features account for $\sim 29 \%$ of the total spectral power in the reconstructed time series.

These two figures give us the times period(s) over which the 9.3 year and 3.8 year oscillatory signals are most prominent. Fig. (4a) shows that the 9.3 year spectral feature is significant throughout the whole period between 1860 and 2010, although it is considerably enhanced between 1910 and 1970, while Fig. (4b) indicates that the 3.8 year feature is enhanced around the 1930's and from about 1985 until 2010.

\subsection{Cross - Correlation}

Fig. (5) shows the summer latitude anomaly of the peak of the STR ( $\mathrm{L}_{\mathrm{SA}}$ in degrees), plotted against the angle between the Earth-Sun axis and the line-of-nodes of the Lunar orbit at perihelion $(\theta$, also measured in degrees). The angle $\theta$ has been used to group the individual data points into bins from $0^{\mathrm{O}}-10^{\mathrm{O}}$, $10^{\mathrm{O}}-30^{\mathrm{O}}, 30^{\mathrm{O}}-50^{\mathrm{O}}, 50^{\mathrm{O}}-70^{\mathrm{O}}$, and $70^{\mathrm{O}}-90^{\mathrm{O}}$. 


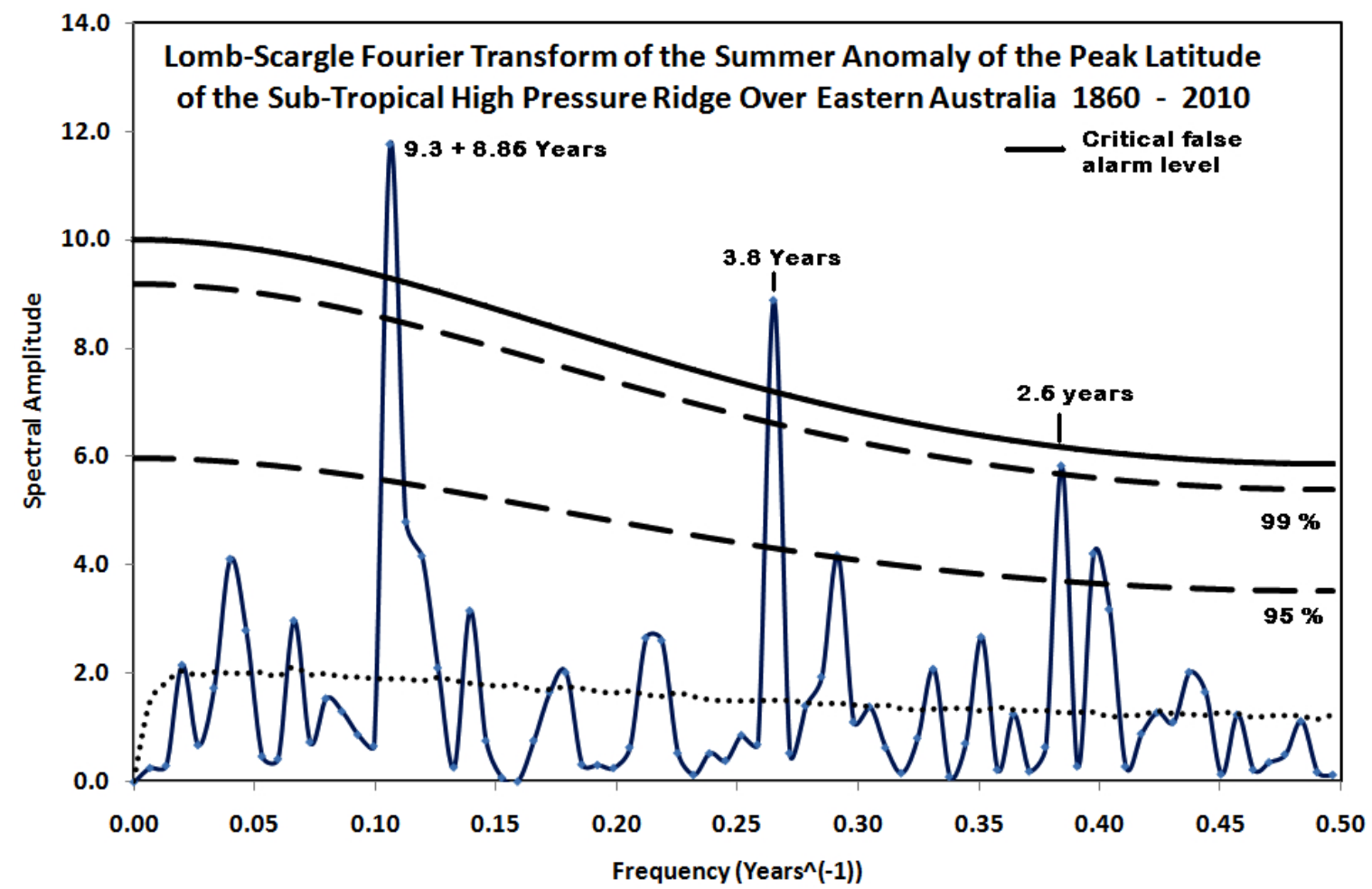

Fig. (3). A Lomb-Scargle periodogram of the $\mathrm{L}_{\mathrm{SA}}$ data set. The spectral amplitude is scaled such that the area under the spectrum is an estimator for the data variance.

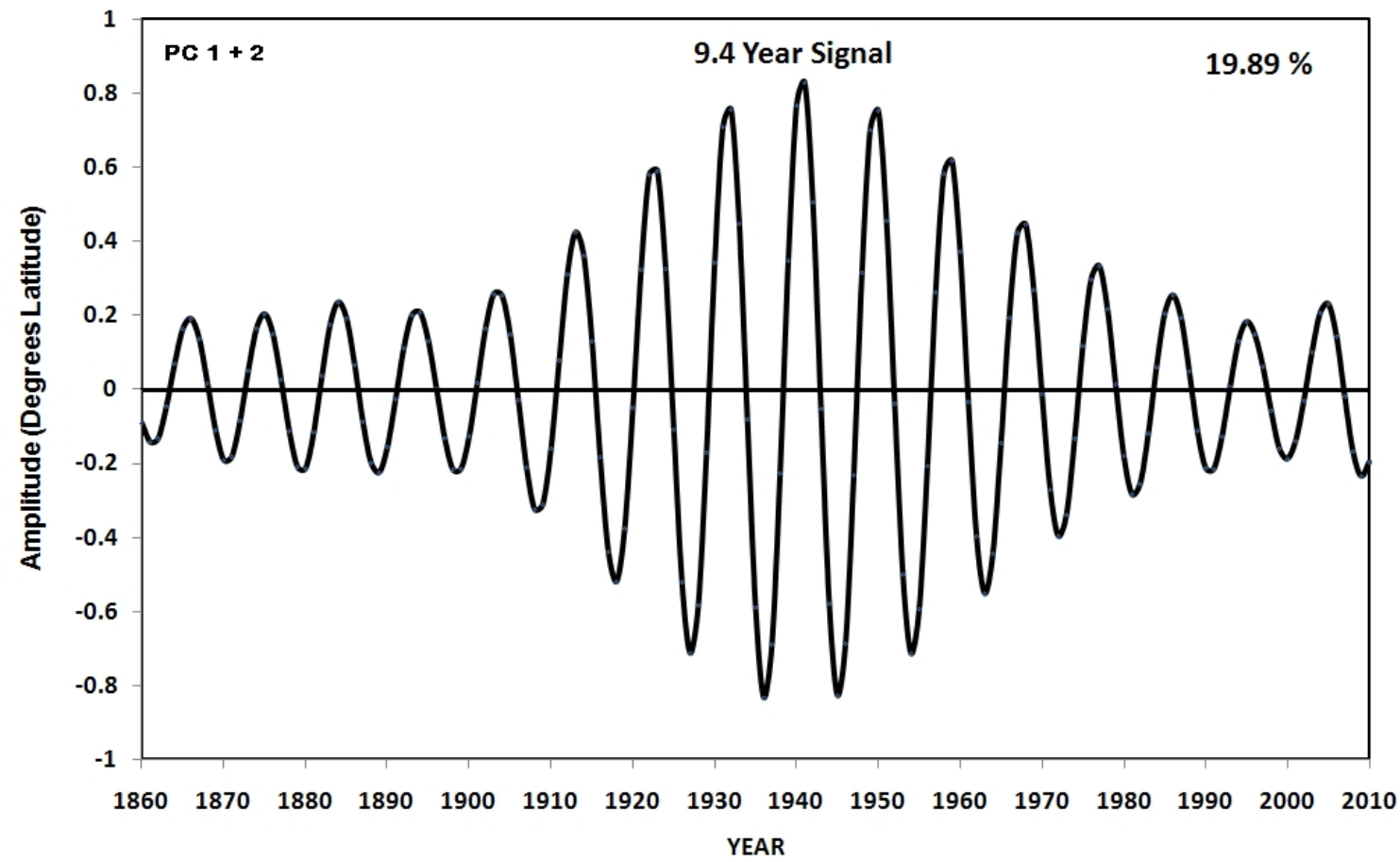

Fig. (4a). The reconstructed time series that uses the first two principal components (PC) of the SSA of the $\mathrm{L}_{\mathrm{SA}}$ time series. PC1 and 2 are associated with the 9.3 year spectral feature in the periodogram of $\mathrm{L}_{\mathrm{SA}}$.

The vertical error bars shown on each data point in Fig. (5) represent the $95 \%$ confidence limits derived from the standard error on the mean for each bin (i.e. $\pm 1.96 \times \sigma / \sqrt{N}$, where $\sigma$ is equal to the standard deviation for the $\mathrm{N}$ data points within each bin). A clear trend is evident between the two variables plotted in Fig. (5), with $\mathrm{L}_{\mathrm{SA}}$ moving away from the Equator as $\theta$ decreases.

The mean data point for each of the bins in Fig. (5), have been used to determine a line-of-best-fit. This fit has then been used to scale $\theta\left(\varphi=0.013 \times \theta-0.347\right.$ where $\left.R^{2}=0.92\right)$ so that $\varphi$ 's mean and variance are comparable to that of the 


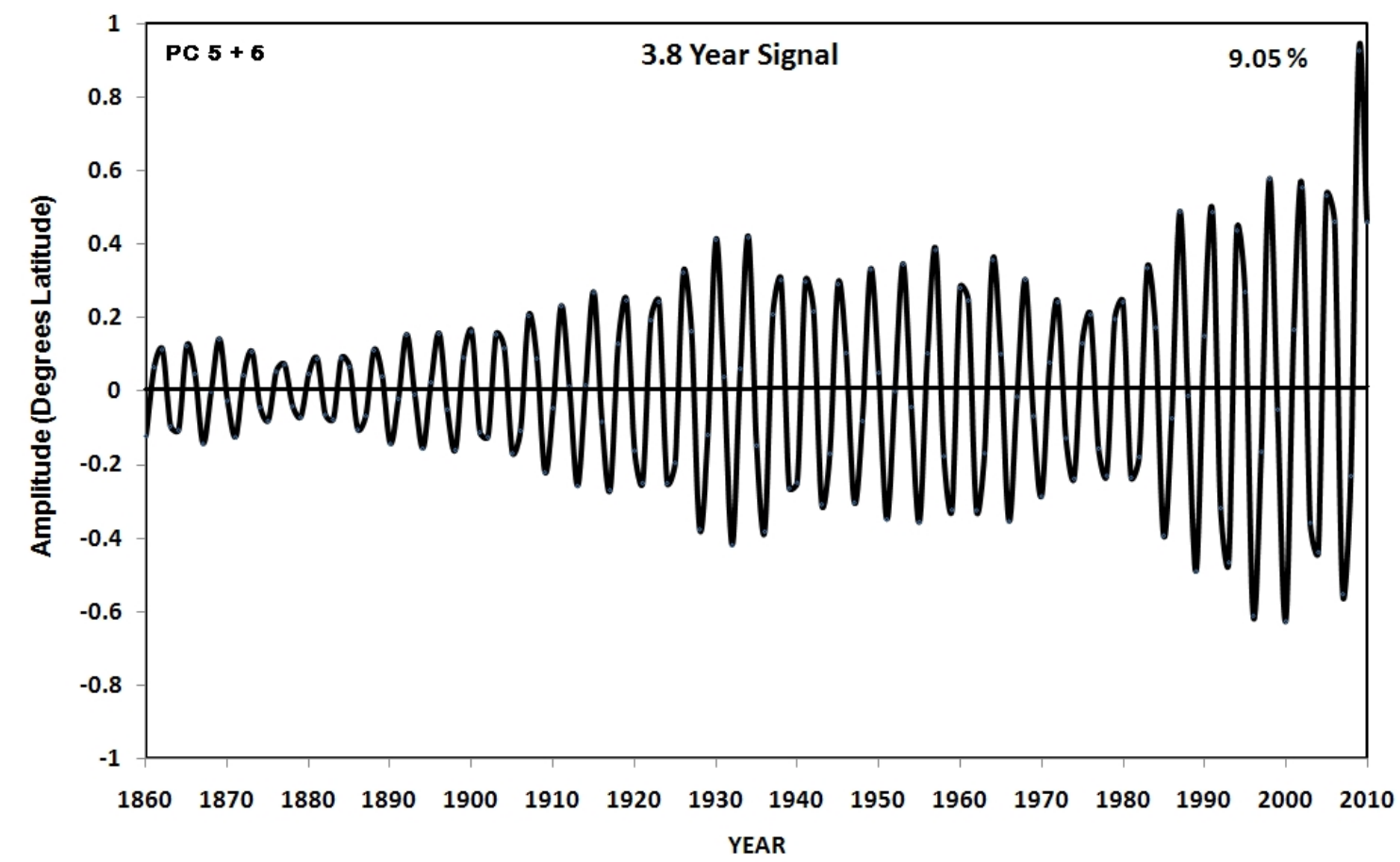

Fig. (4b). The reconstructed time series that uses the $5^{\text {th }}$ and $6^{\text {th }}$ principal components (PC) of the SSA of the $\mathrm{L}_{\mathrm{SA}}$ time series. PC5 and 6 are associated with the 3.8 year spectral feature in the periodogram of $\mathrm{L}_{\mathrm{SA}}$.

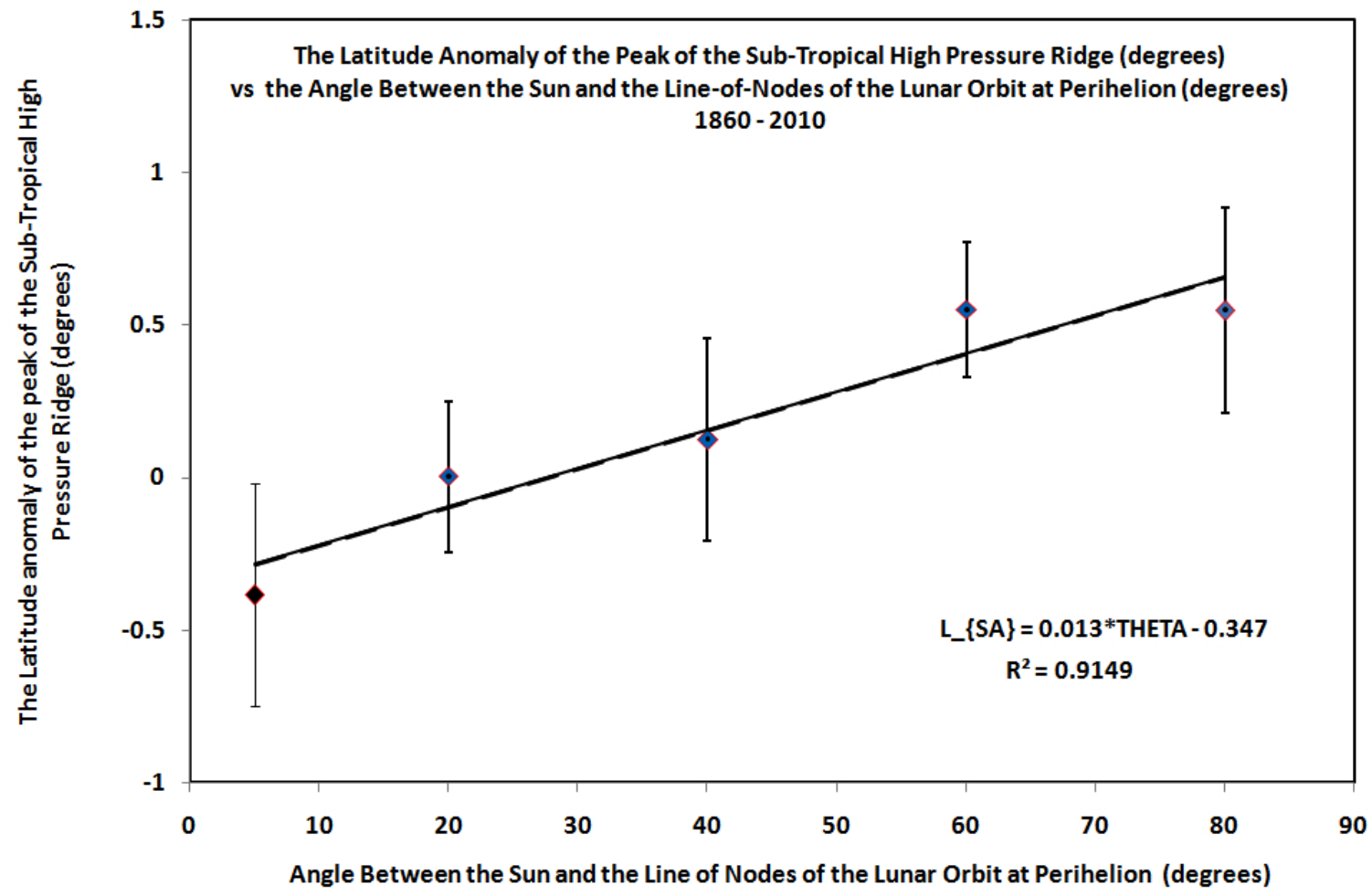

Fig. (5). The summer latitude anomaly of the peak of the STR ( $\mathrm{L}_{\mathrm{SA}}$ in degrees), plotted against the angle between the Earth-Sun axis and the line-of-nodes of the Lunar orbit at perihelion ( $\theta$, also measured in degrees). The angle $\theta$ has been used to group the individual data points into bins from $0^{\mathrm{O}}-10^{\mathrm{O}}, 10^{\mathrm{O}}-30^{\mathrm{O}}, 30^{\mathrm{O}}-50^{\mathrm{O}}, 50^{\circ}-70^{\mathrm{O}}$, and $70^{\mathrm{O}}-90^{\circ}$.

$\mathrm{L}_{\mathrm{SA}}$ time series. It is important to note that the time series for $\varphi$ has been calculated using the observed values of $\theta$ for each year.

In order to establish a strong link between the 9.3 year spectral feature and the period of the draconic seasonal peak tides, we need to cross correlate the $\mathrm{L}_{\mathrm{SA}}$ time series with $\varphi$ and show that the 9.3 year spectral signal is in phase with the draconic seasonal peak tidal cycle.

Fig. (6) shows a plot of the normalized cross-correlation function for $\mathrm{L}_{\mathrm{SA}}$ and $\varphi$, where both time series have been 


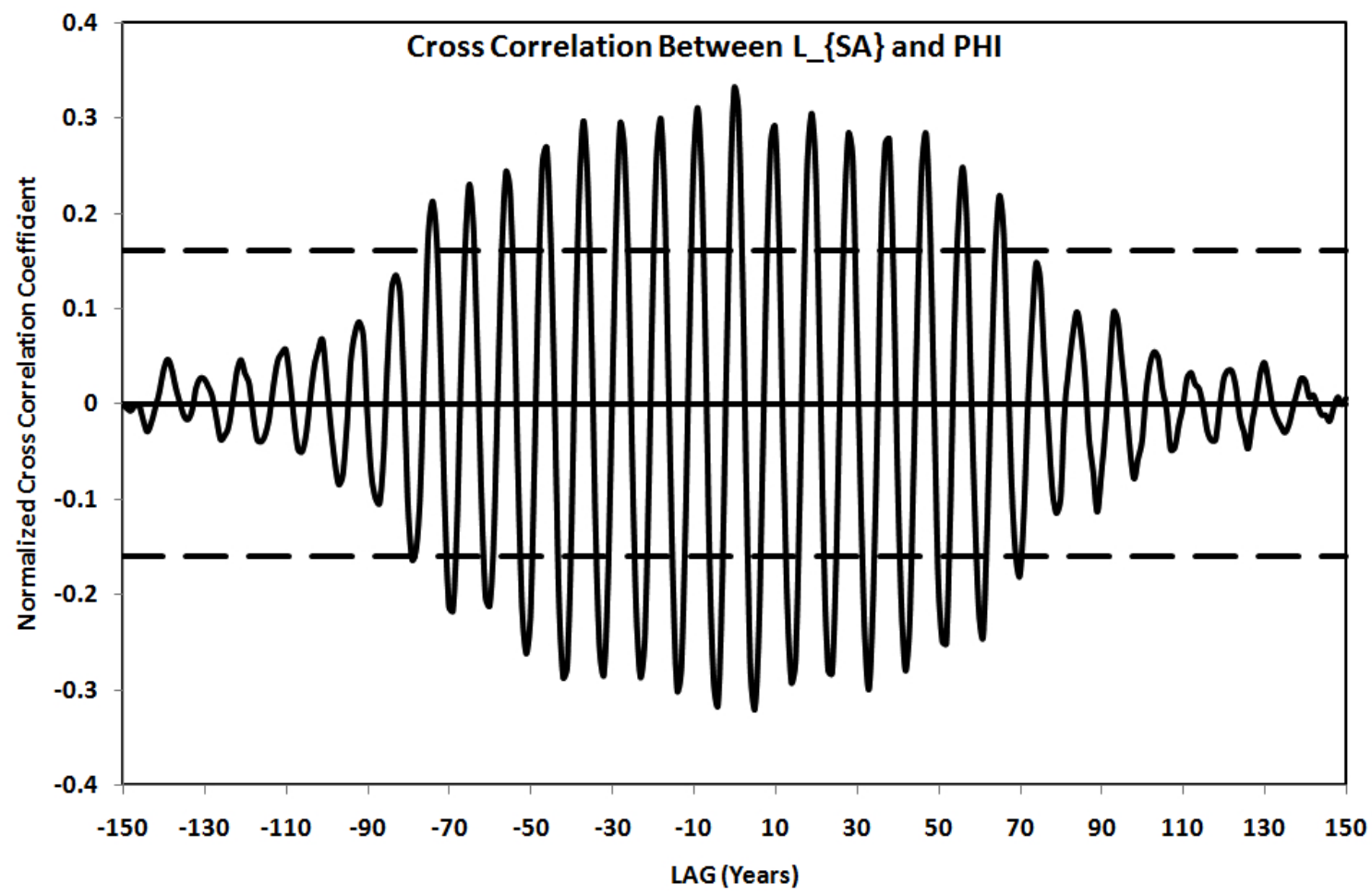

Fig. (6). This figure shows a plot of the normalized cross-correlation function for $\mathrm{L}_{\mathrm{SA}}$ and $\varphi$, where both time series have been shifted to a zero mean. Horizontal dotted lines are superimposed on this figure to indicate the $95 \%$ confidence levels for the cross correlation coefficient. The norm $=1$ option of the XCORR worksheet function in the DADiSP 2002 software package has been used to normalize the crosscorrelation function.

shifted to a zero mean (N.B. a cross-correlation between $\mathrm{L}_{\mathrm{SA}}$ and $\theta$ would produce the same result as the current cross-correlation, since the linear scaling of $\theta$ has no effect upon the phase shift).

Horizontal dotted lines are superimposed on Fig. (6) to indicate the $95 \%$ confidence levels (i.e. $\pm 1.96 / \sqrt{N}$ where $\mathrm{N}$ is the number of data points) for the cross correlation coefficient that rules out the null hypothesis that at least one of the times series is white noise. The resultant plot indicates that the cross correlation coefficient for $\mathrm{L}_{\mathrm{SA}}$ and $\varphi$ is significant, and that the observed 9.3 year periodic signal in the $\mathrm{L}_{\mathrm{SA}}$ time series is in-phase with the 9.3 year period of the draconic seasonal peak tidal cycle (as measured by $\varphi$ ).

Fig. (7) shows $\mathrm{L}_{\mathrm{SA}}$ (measured in degrees) placed in bins of the number of days (to the nearest full day) that New/Full Moon is from Perihelion in the following year (i.e. ST - 1). As with Fig. (5), the vertical error bars shown on each data point in Fig. (7) represent the $95 \%$ confidence limits derived from the standard error on the mean for each bin. Again, a clear trend is evident between the two variables plotted in Fig. (7), with $\mathrm{L}_{\mathrm{SA}}$ moving towards the Equator as (ST - 1) decreases.

The mean data point for each of the bins in Fig. (7) has been used to determine a line-of-best-fit. This fit has then been used to scale $(\mathrm{ST}-1)(\psi=-0.121 \times(\mathrm{ST}-1)+0.680$ where $\left.\mathrm{R}^{2}=0.83\right)$ so that $\psi$ 's mean and variance are comparable to that of the $\mathrm{L}_{\mathrm{SA}}$ time series. It is important to note that the time series $\psi$ has been calculated using the observed values of $(\mathrm{ST}-1)$ for each year.
As with the 9.3 year spectral feature, a stronger link between the 3.8 year feature and the period of the spring tide seasonal peak tides is established if the cross correlation between the $\mathrm{L}_{\mathrm{SA}}$ time series and $\psi$ shows that 3.8 year spectral signal is in-phase with the spring tide seasonal peak tidal cycle.

Fig. (8) shows a plot of the normalized cross-correlation function for $\mathrm{L}_{\mathrm{SA}}$ and $\psi$, where both time series have been shifted to a zero mean. Horizontal solid lines are superimposed on this figure at values of \pm 0.16 to indicate the $95 \%$ confidence levels (i.e. $\pm 1.96 / \sqrt{N}$ where $\mathrm{N}$ is the number of data points) for the cross correlation coefficient that rules out the null hypothesis that at least one of the times series is white noise.

It is evident from Fig. (8) that the cross correlation between $\mathrm{L}_{\mathrm{SA}}$ and $\psi$ is significant and that the observed 3.8 year periodic signal in the $\mathrm{L}_{\mathrm{SA}}$ time series is retarded by one year in phase (i.e. one year behind) compared to the 3.8 year period of the spring tide seasonal tidal cycle. Interestingly, Fig. (8) shows that there is a correlation in signal between parts of the $\mathrm{L}_{\mathrm{SA}}$ time series that are separated by 19 years $(=$ $5 \times 3.8$ years), a period of time that is equal to the Lunar Metonic cycle.

\section{DISCUSSIONS AND CONCLUSIONS}

This study has attempted to extend the pioneering work of Li et al. [5] by looking for evidence of an atmospheric lunar tidal signal in the variability of the peak latitude of the summer anomaly of the subtropical high pressure ridge 


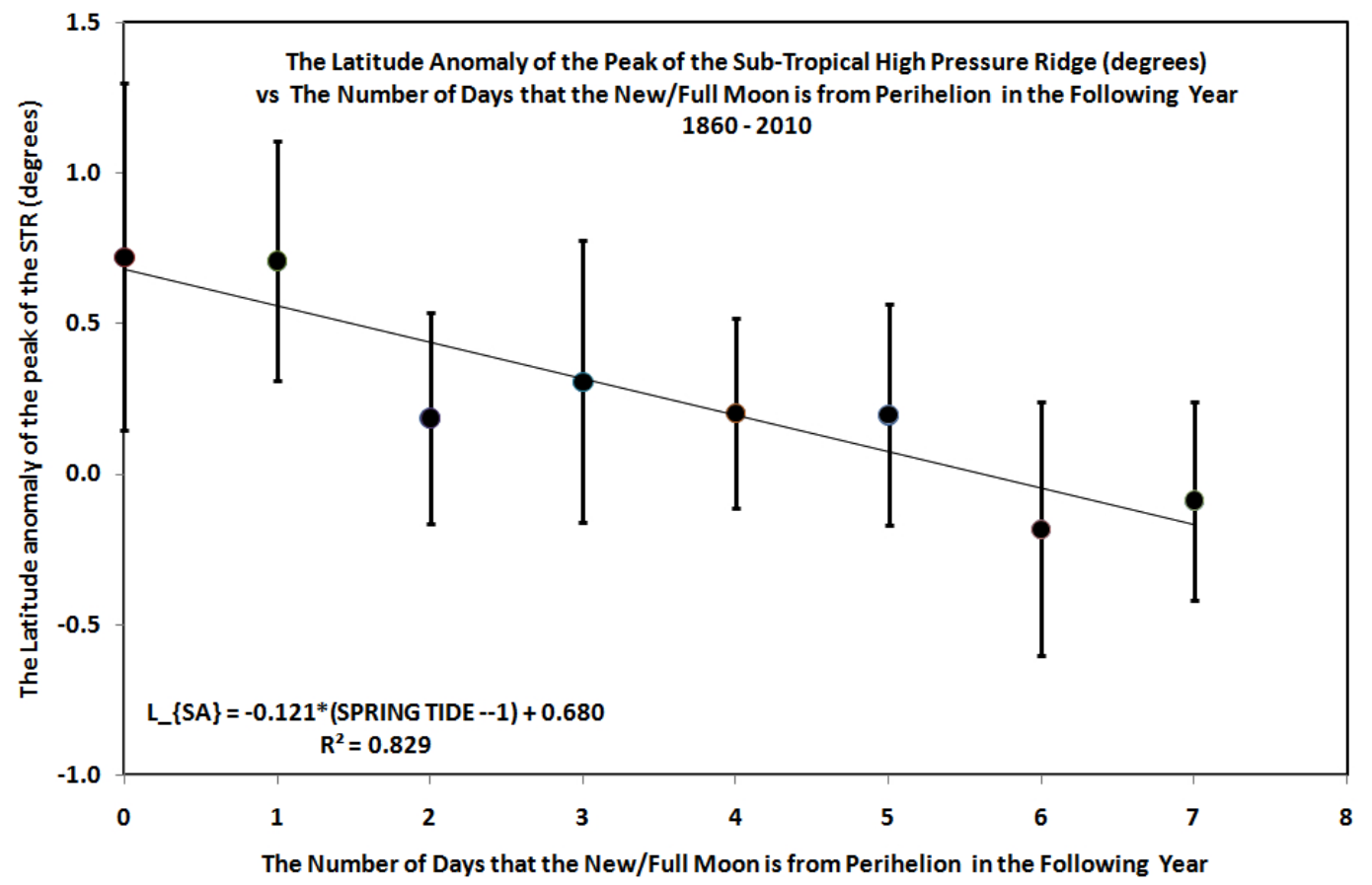

Fig. (7). The Summer latitude anomaly of the peak of the STR ( $\mathrm{L}_{\mathrm{SA}}$ in degrees) placed in bins of the number of days (to the nearest full day) that New/Full Moon is from Perihelion in the following year (i.e. ST - 1).

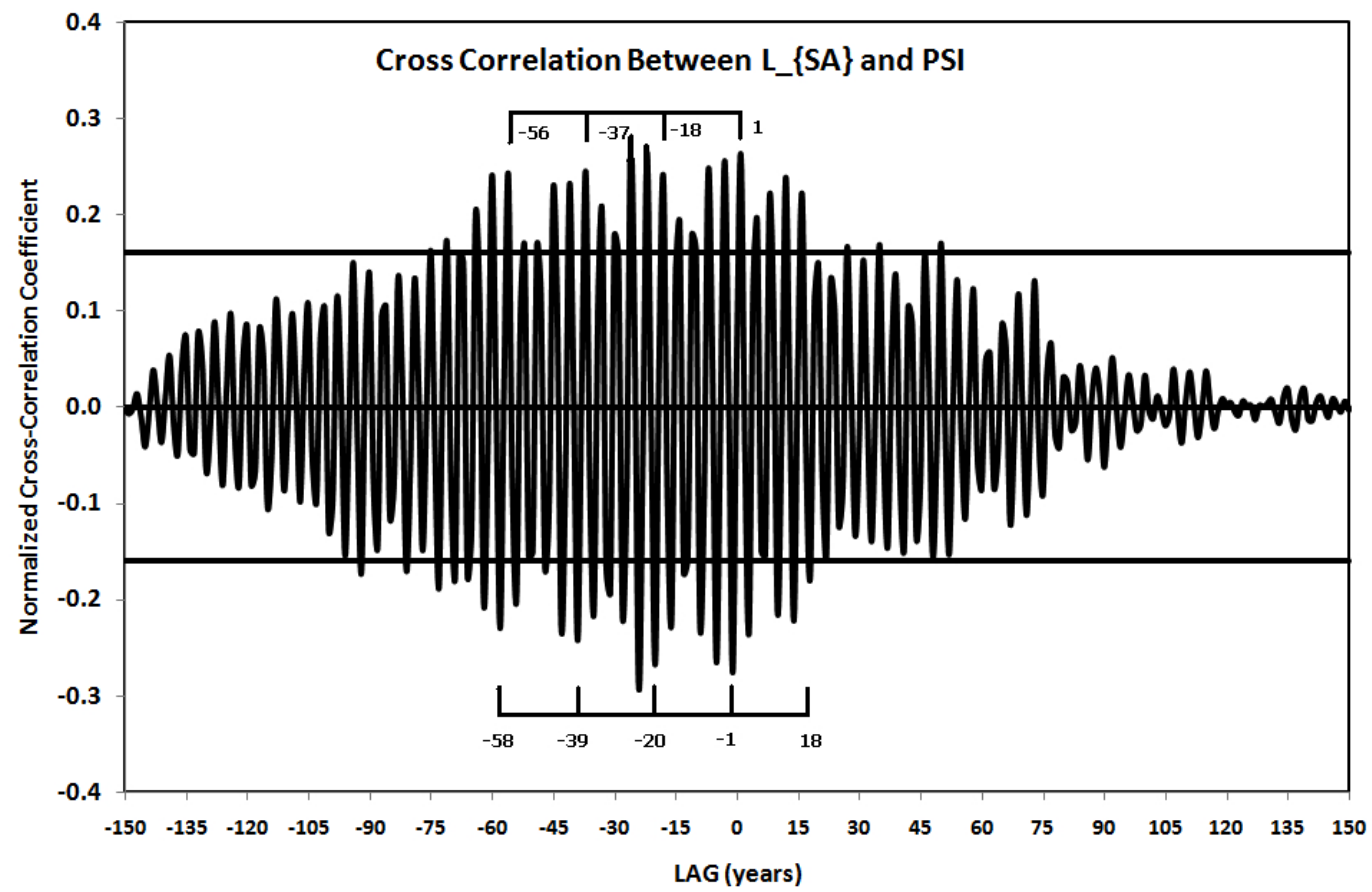

Fig. (8). This figure shows a plot of the normalized cross-correlation function for $\mathrm{L}_{\mathrm{SA}}$ and $\psi$, where both time series have been shifted to a zero mean. Horizontal solid lines are superimposed on this figure to indicate the $95 \%$ confidence levels for the cross correlation coefficient. The norm $=1$ option of the XCORR worksheet function in the DADiSP 2002 software package has been used to normalize the crosscorrelation function.

(STR) over Eastern Australia, on inter-annual to decadal timescales (i.e. from 2 to 20 years).

Two separate methods were used to infer the potential periods for the atmospheric lunar tidal signal that might be observed in the atmospheric pressure record.
The first method assumes that lunar-tidal forces act independently of the other forcing factors that produce significant long-term variations in atmospheric pressure. It predicted that you should see long-term periodicities in the pressure records that match periodicities of the most extreme peak lunar tides. This analysis identified four potential long- 
term periods for the atmospheric lunar tidal signal at periods of 3.01, 6.01, 9.02 and 18.03 (tropical) years. None of these periods were observed in the $\mathrm{L}_{\mathrm{SA}}$ time series.

The second method assumed that if lunar tides do play a role in influencing atmospheric pressure, it was most likely one where the tidal forces act in "resonance" with the changes caused by the far more dominant solar-driven seasonal cycles. In this case, it is not so much in what years do the lunar tides reach their maximum strength, but whether or not there are peaks in the strength of the lunar tides that re-occur at the same time within the annual seasonal cycle. This simple "resonance" model identified three potential long-term periods for the atmospheric lunar tides matching those of the 3.8 year peak spring tides, the 8.85 year perigean spring tides, and the 9.3 year draconic spring tides.

A Lomb-Scargle periodogram of the peak latitude of the summer anomaly of the STR over Eastern Australia $\left(\mathrm{L}_{\mathrm{SA}}\right)$ shows that there are significant peaks in this times series at $9.4(+0.4 /-0.3)$ and $3.78( \pm 0.06)$. The 9.4 year peak is consistent with the 9.3 year period of the Draconic spring tidal cycle and the 3.8 year peak is consistent with the 3.8 year period of the peak spring tidal cycle. Singular spectrum analysis of the $\mathrm{L}_{\mathrm{SA}}$ time series shows that the 9.4 year spectral feature is significant throughout the whole period between 1860 and 2010 while the 3.8 year feature is enhanced around the 1930's and from about 1985 until 2010.

A linear trend is established between $\mathrm{L}_{\mathrm{SA}}$ and $\varphi$ where $\varphi$ $=0.013 \theta-0.347$ and $\theta$ is the angle between the Earth-Sun axis and the line-of-nodes of the Lunar orbit at perihelion. $\theta$ is an indicator of the strength of the 9.3 year Draconic spring tides. The form of this trend is such that $\mathrm{L}_{\mathrm{SA}}$ moves away from the Equator as $\theta$ decreases.

A second linear trend is established between $\mathrm{L}_{\mathrm{SA}}$ and $\psi$ where $\psi=-0.121(\mathrm{ST}-1)+0.680$ and $(\mathrm{ST}-1)$ is the number of days (to the nearest full day) that a New/Full Moon is from Perihelion, in the following year. The number of days that a New/Full Moon is from Perihelion (i.e. ST) is an indicator of the strength of the 3.8 year peak spring tides. The form of this second trend is such that $\mathrm{L}_{\mathrm{SA}}$ moves towards the Equator as $(\mathrm{ST}-1)$ decreases.

A cross-correlation between $\mathrm{L}_{\mathrm{SA}}$ and $\varphi$ shows that the 9.3 year periodic signal in the $\mathrm{L}_{\mathrm{SA}}$ time series is in-phase with the 9.3 year draconic spring tidal cycle. Similarly, a crosscorrelation between $\mathrm{L}_{\mathrm{SA}}$ and $\psi$ shows that the 3.8 year periodic signal in the $\mathrm{L}_{\mathrm{SA}}$ time series is retarded by one year in phase (i.e. one year behind) compared to the 3.8 year peak spring tidal cycle.

This study shows that if you control for the seasonal (solar) effects upon the peak latitude anomaly of the SubTropical high pressure ridge over Eastern Australia, by limiting your analysis to the summer months, it is possible to discern the periodicities in the pressure ridge's peak latitude that are caused by lunar tidal forces acting over inter-annual to decadal timescales.

\section{ACKNOWLEDGMENTS}

The author would like to thank Dr. Norman Treloar and Prof. Nikolay Sidorenkov for inspiring the research done in this paper through their pioneering investigations of the influence of lunar tides upon climate. In addition, the author would like to thank Christopher Game and Paul Vaughan for the advice and guidance they gave in the planning and preparation of this paper.

\section{CONFLICT OF INTEREST}

No outside funding was used to conduct this work. No conflicts of interest are involved.

\section{REFERENCES}

[1] Chapman S, Lindzen RS. Atmospheric tides: thermal and gravitational. D. Reidel Publishing Company: Dordrecht, Holland 1970.

[2] Lindzen RS. Dynamics in atmospheric physics. Cambridge University Press: Cambridge 2005.

[3] Li G. 27.3-day and 13.6-day atmospheric tide and lunar forcing on atmospheric circulation. Adv Atmos Sci 2005; 22(3): 359-74.

[4] Li G, Zong H. 27.3-day and 13.6-day atmospheric tide. Science in China (D) 2007; 50(9): 1380-95.

[5] Li G, Zong H, Zhang Q. 27.3-day and average 13.6-day periodic oscillations in the earth's rotation rate and atmospheric pressure fields due to celestial gravitation forcing. Adv Atmos Sci 2011; 28(1): 45-58.

[6] Defraigne P, Smits I. Length of day variations due to zonal tides for an inelastic earth in non-hydrostatic equilibrium, Geophys J Int 1999; 139: 563-72.

[7] Yoder CF, Williams JG, Parke ME. Tidal variations of earth rotation. J Geophys Res 1981; 86(B2): 881-91.

[8] Varga P, Gambis D, Bizouard Ch, Bus Z, Kiszely M. Tidal influence through LOD variations on the temporal distribution of earthquake occurrences. Journées 2005 - systèmes de référence spatio - temporels, warsaw, Space Research Centre Polish Academy of Sciences 2005.

[9] Keeling CD, Whorf TP. Possible forcing of global temperature by the oceanic tides. Proc Natl Acad Sci USA 1997; 94: 8321-8.

[10] Standish EM, Williams JC. Orbital ephemerides of the sun, moon and planets (PDF). International Astronomical Union Commission 4: Ephemerides 2010.

[11] McCarthy DD, Seidelmann PK. Time from earth rotation to atomic physics. Weinhein: Wiley-VCH Verlag GmbH \& Co KGaA 2009.

[12] Allen CW, Cox AN. Allen's astrophysical quantities: 4th ed. New York: Springer-Verlag 2000.

[13] Chapront-Touzé M, Chapront J. Lunar tables and programs from 4000 B.C. to A.D. 8000. William-Bell: Richmond, Virginia 1991.

[14] Meeus J. Extreme perigees and apogees of the moon. Sky Telescope 1981; 62; 110-111

[15] Wood FJ. Tidal dynamic-coastal flooding, and cycles of gravitational force. D. Reidel: Dordrecht and Boston 1986.

[16] Pittock AB. Rainfall and the general circulation. Int. Conference in Weather Modification, Canberra 1971.

[17] Coughlan MJ. Recent variations in annual mean maximum temperatures over Australia. Q J R Met Soc 1979; 105: 707-19.

[18] Thresher RE. Solar correlates of southern hemisphere mid-latitude climate variability. Int J Climatol 2002; 22: 901-15.

[19] Pittock, AB. Global meridional interactions in stratosphere and troposphere. Q J R Met Soc 1973; 99: 424-37.

[20] Allan RJ, Ansell TJ. A new globally complete monthly historical mean sea level pressure data set (HadSLP2): 1850 - 2004. J Climate 2006; 19: 5816-42.

[21] www.hadobs.org, hadSLP2r, accessed: Mar 2011

[22] DADisp 2002: software program - DSP Development Corporation 2006, www.dadisp.com

[23] Microsoft Excel software package - Microsoft Office 2007.

[24] Williams AJ, Stone RC. An assessment of relationships between the Australian subtropical ridge, rainfall variability, and highlatitude circulation patterns. Int J Climatol 2009; 29: 691-709.

[25] Lomb NR, Least-squares frequency analysis of unequally spaced data. Astrophys Space Sci 1976; 39: 447-62.

[26] Scargle JD. Studies in astronomical time series analysis. II. statistical aspects of spectral analysis of unevenly spaced data. Ap J 1982; 263: 835-53.

[27] Scargle JD. Studies in astronomical time series analysis. II. fourier transforms, autocorrelation functions, and cross-correlation functions of unevenly spaced data. Ap J 1989; 343: 874-87. 
[28] Schulz M, Mudelsee M. REDFIT: estimating red-noise spectra directly from unevenly spaced paleoclimatic time series. Comp Geosci 2002; 28: 421-6.
[29] Caterpillarv1.0 1997: gus@vega.maths.spbu.ru.

[30] Thomson DJ. Time series analysis of Holocene climate data. Philosophical Trans R Soc Lond Ser A 1990; 330: 601-16.

(C) Ian R.G. Wilson; Licensee Bentham Open.

This is an open access article licensed under the terms of the Creative Commons Attribution Non-Commercial License (http: //creativecommons.org/licenses/by$\mathrm{nc} / 3.0 /$ ) which permits unrestricted, non-commercial use, distribution and reproduction in any medium, provided the work is properly cited. 